\title{
Design and optimization of a rubber-bitumen blend in preparation for a rubberized-asphalt road trial in the state of Kuwait
}

\author{
Salah E. Zoorob ${ }^{1, *}$, Suad K. Al-Bahar ${ }^{1}$, and Saud F. Al-Otaibi ${ }^{1}$ \\ ${ }^{1}$ Construction and Building Materials Program, Kuwait Institute for Scientific Research, P.O. Box \\ 24885 Safat, 13109 Kuwait.
}

\begin{abstract}
In this paper, test results are presented from laboratory trials of blending discarded tire derived crumb rubber with 60/70pen grade Kuwaiti bitumen. The virgin bitumen, in addition to $15 \%$ and $18 \%$ crumb rubber blends were tested for PG grading and multiple stress creep recovery at temperatures in the range from $40^{\circ} \mathrm{C}$ to $70^{\circ} \mathrm{C}$. Elastic recovery and nonrecoverable creep compliance showed optimum performance at $18 \%$ rubber content. The optimized rubberized-bitumen blend was next converted into a pelletized form which was then used as a partial substitute to the conventional bitumen in the manufacture of continuously graded hot mix asphalts at various binder contents. The rubberized-bitumen pellet contents trialed were $2.5 \%, 3.0 \%$ and $3.5 \%$ by mass of mix. The Marshall method of mix design was used to assess the various mixes and the overall volumetric, stability and flow results, though satisfactory, was not entirely sufficient to aid in the selection of the best performing formulation.
\end{abstract}

\section{Introduction}

The State of Kuwait's total length of main paved roads is slightly in excess of $7518 \mathrm{~km}$ (equivalent to an area of $87,411,674 \mathrm{~km}^{2}$ ). The network suffers from a number of asphalt pavement distress mechanisms including; fatigue cracking, rutting, bleeding, potholes and raveling. The primary causes vary from one site to the other and include; incorrect bitumen grade, excessive binder oxidation, incorrect mix designs and binder contents, high traffic loading, settlement failure in the lower layers, inadequate compaction, very high climatic temperatures, lack of high-quality local mineral aggregates, etc.

For many decades, the only bitumen that was available for use in road construction in Kuwait has been a standard 60/70pen grade bitumen which in rheological terms is equivalent to a US Performance Grade PG 64-10. The designation PG 64-10 indicates that the bitumen will "in theory" satisfy performance requirements for a temperature zone that has an average maximum consecutive 7-day pavement temperature of less than $64^{\circ} \mathrm{C}$ and a minimum temperature greater than $-10^{\circ} \mathrm{C}$. In recent years, Kuwait has been experiencing exceptionally hot summers, as a notable example, on the $20^{\text {th }}$ July 2017 the maximum air temperature in Kuwait City was reported officially at $54^{\circ} \mathrm{C}$. Figure 1 shows an example of

*Corresponding author: salah.zoorob@googlemail.com 
the relation between air temperature and recorded temperature at a depth of $20 \mathrm{~mm}$ in pavements in Saudi Arabia, Kuwait and the UAE [1,2].

The Ministry of Public Works (MPW) in the State of Kuwait has responsibility for maintaining the serviceability and ride quality of the entire road network. In order to improve life cycle costing and reduce the frequency of road maintenance operation, the MPW in 2017 advised all asphalt plant operators in Kuwait to upgrade their existing hot mix asphalt (HMA) production and laboratory facilities to accommodate the handling and incorporation of polymer modified bitumens (PMBs).

In parallel, the Construction \& Building Materials Program at the Kuwait Institute for Scientific Research (KISR) has initiated an investigation into the use of waste tire derived crumb rubber for the manufacture of high-performance rubberized-HMA using locally sourced aggregates and standard Kuwaiti bitumen. It is intended that a pilot road surfacing trial will be subsequently conducted using rubberized-HMA on a highly trafficked road in Kuwait as part of routine maintenance operations. This road trial will be designed to assess the benefits of rubberized HMA technology, in particular with respect to alleviating rutting, fatigue cracking and moisture induced stripping, and observe any noticeable impact or shortfalls during HMA production, transport, laying and compaction operations.

It is interesting to note that in Kuwait the largest waste tyre dump holds approximately 28 million whole tyres in a chaotic fashion whereby some of the old burial pits are in excess of $20 \mathrm{~m}$ deep. The government has taken progressive steps in 2017 to recover the buried tyres and rough cut them into large pieces which are being transported and safely stored at an alternative site. Meanwhile, 5000 to 6000 discarded tyres continue to be added to the dump per day. The large quantities of discarded waste tyre crumb rubber that can be utilized in bitumen modification should lead to positive local environmental impact. For example, to replace only the wearing course (i.e. $5 \mathrm{~cm}$ thick layer) of a single lane on a damaged road using rubberized-HMA would consume approximately 4.5 tons of crumb rubber per $1 \mathrm{~km}$ length of road (equivalent to 100 discarded truck tyres or 500 car tyres).

\section{Background to rubberized hot mix asphalt}

Rubberized-HMA is a process of blending crumb rubber produced from recycled waste tires into hot bitumen (wet process) to produce an extremely resilient, high performance, modified binder (rubberized-bitumen) that is used in road paving operations. The original American Society of Testing and Materials (ASTM D6114, now withdrawn) definition stipulates that the rubber component in the rubber-bitumen blend must be at least $15 \%$ by weight of the total blend and must have reacted in the hot bitumen sufficiently to cause swelling of the rubber particles.

The rubberized-HMA process originated in the United States in the late 1960s, and since that time a large collection of historical data has been amassed that highlights crumb rubber's enhancement of mixture performance including; improved rutting resistance, thermal cracking resistance, reflective crack resistance and resistance to load-induced fatigue cracking. Other benefits reported include reduction in the frequency of maintenance operations, a smooth ride, good skid resistance, and noise reduction [3-5]. Lo Presti [6] presented an excellent review of the existing technologies and specifications related to the production, handling and storage of rubber modified bitumens and their current applications within road asphalt mixtures.

A standard practice guide, published in 2012 by the Rubber Pavement Association (RPA), provides an overview of all the necessary basic information regarding the design and use of rubberized-HMA [7]. The guide covers every aspect of the rubberized-HMA mix production process including the types and preferred gradations of crumb rubber, rubber-bitumen blending techniques, laboratory testing requirements, a variety of potential 
road applications, performance requirements, etc. At minimum, the following laboratory tests are recommended by the RPA guide for characterizing rubber-bitumen blends during the design stage: a- Rotational viscosity $\left(1.5-4.0\right.$ Pa.s) at $177^{\circ} \mathrm{C}$, b- Resilience at $25^{\circ} \mathrm{C}$ (min. 30\%) (ASTM D5329), c- Ring and Ball Softening point (min. $55^{\circ} \mathrm{C}$ ) (ASTM D36), dPenetration at $4^{\circ} \mathrm{C}(\mathrm{min} .10 \mathrm{dmm})$ at $60 \mathrm{sec}$. loading time using a $200 \mathrm{~g}$ needle (ASTM D5).

Factors that impact the cost of rubberized-HMA include: established usage of the product, capital costs, increased material costs, and increased operational costs. Additional factors that influence individual project costs are project size, patent royalty fees, and the contractor's "fear factor" associated with unknown impacts on plant processes such as increased fuel costs for higher temperatures, plant/equipment damage, downtime changing between rubber modified and unmodified jobs [8].

The actual mixing process of the rubberized-HMA at the hot mix asphalt plant is the same as for conventional HMA [9]. Components that need to be added at the asphalt plant are; rubber storage, rubber feed, heated blending tanks (at approx. $190^{\circ} \mathrm{C}$ ), and (optional) a heated reaction tank (at approx. $177^{\circ} \mathrm{C}$ ). A separate connection from the plant's standard bitumen feed is made to the pug mill mixer. This allows the plant to produce other mixes without unnecessary delays. Rubber storage requirements will depend upon the method used for adding the rubber. Bagged rubber usually comes in 10-15 $\mathrm{kg}$ paper bags on pallets which are added to the tanks manually. Bulk rubber can be stockpiled (covered) and fed into the tanks via loading buckets, hopper, and conveyer systems [8].

Hauling of the rubberized-HMA is the same as for conventional HMA except that soapy water or a silicone emulsion should be used for a releasing agent. Diesel or solvents should not be used. This difference should result in no cost differential between two mix types. The placement and compaction of rubberized-HMA are generally the same as for conventional HMA, though the placement temperature for rubberized-HMA is somewhat higher than for conventional HMA, and furthermore, pneumatic rollers cannot be used for compacting rubberized-HMA. Instead, vibratory (for breakdown rolling) and static steel wheel rollers should be used [9].

In the United States of America, there are currently 25 states which legislate the use of rubberized-HMA for improving road performance and for minimizing the frequency of road maintenance operations and related costs. In addition, rubberized-HMA has been successfully used in Portugal, South Africa, Spain, China, Poland, Saudi Arabia, Mexico, Russia, Brazil, Puerto Rico, Italy, and the Czech Republic [7].

The experimental data presented in this paper are the results of preliminary laboratory trials conducted at Kuwait Institute for Scientific Research in collaboration with Phoenix Industries USA, to evaluate the compatibility of the locally produced discarded tire derived crumb rubber with standard Kuwaiti 60/70pen bitumen and the effects of rubber content on rheological properties of the resultant rubber-bitumen blend.

\section{Materials used and sample preparation}

Samples of a conventional air blown 60/70pen Kuwaiti bitumen (pen. $=69$ dmm, S.P. $=$ $50.2^{\circ} \mathrm{C}$ ) and a PG 67-22 bitumen produced by Associated Asphalt (supplier to paving contractors in the Southeastern USA) sourced from Inman S.C. terminal, were used in this investigation.

In the first stage, rubber-bitumen blends were produced in the laboratory using a high shear mixer at $15 \%$ and $18 \%$ ground tyre rubber content (100\% passing $0.6 \mathrm{~mm})$. This level of crumb fineness is necessary when dense graded rubberized asphalt mixes are being designed as opposed to coarser gradations that may be used with a gap or open graded HMA [10]. The blends were produced at $180^{\circ} \mathrm{C}, 3500 \mathrm{rpm}$ with minimal high shear time (approx. 20 minutes for a $5 \mathrm{~L}$ blend) followed by 45 minutes agitation at $250 \mathrm{rpm}$. 
The neat bitumens together with the $15 \%$ and $18 \%$ rubber-bitumen blends were subsequently characterized using a dynamic shear rheometer (DSR) for PG grading and Multiple Stress Creep Recovery (MSCR) tests.

To produce the rubber-bitumen blends on a large scale, a model AR150 rubber-bitumen blending unit (Phoenix Industries LLC) with a production rate of up to 15 blended binder tons per hour was used. The unit comprises of a control house, a crumb rubber hopper and a series of pumps and augers up to a blending vessel that the binder is pumped into. The neat bitumen and crumb rubber are combined in a blending pot at $3500 \mathrm{rpm}$ and immediately pumped into a reaction tank. The reaction tank, or holding tank, is a second portable unit comprising of a 30,000 gallon tank split into two halves. In that tank, the rubber reacts with the virgin bitumen and the mix is held for a specified period of time while it cures. The tank is heated and has agitators at the bottom and mid-level so that the material rotates from the bottom up to the top and then back down the sides in one continuous motion [11].

The AR150 unit is designed so that no rubber digestion by shear occurs during the blending process. As far as practicable, the rubber crumb particles are kept intact with no significant reduction in particle size. The blending unit is designed not to produce a "smooth binder like a PMB", rather the binder in the Phoenix process is "lumpy" with rubber particles kept in suspension in the bitumen and clearly visible if the binder is thinly spread on a flat surface. Hence the process is not a high shear/milling type process, rather it is a vigorous dispersion process. The "reaction tank" keeps the blend highly agitated with forced circulation. It was also noted that the tank is divided into 2 compartments, in the first part the crumb is blended with the bitumen, and in the second compartment the blended binder is kept in a state of high agitation. Normally, blend agitation must be maintained right up to the point of adding to the hot aggregate in the asphalt plant, otherwise, settlement of the crumb rubber particles becomes inevitable [11].

For this trial, ground tyre rubber was first blended (high-speed mixing) with Inman PG 67-22 grade bitumen at $190^{\circ} \mathrm{C}$ and subsequently agitated in a blend tank for 45 to 60 minutes at $177^{\circ} \mathrm{C}$. In an ideal scenario, the optimum proportion of truck tyres in the crumb should preferably not exceed $40 \%$, the remainder being car tyre derived crumb. Based on binder characterization test results, including PG grading and multiple stress creep recovery tests, a final rubber-bitumen blend was produced using the AR150 unit (at 18\% crumb rubber content). The $18 \%$ hot blend was next introduced into a pelletizing machine (a Phoenix Industries patented process) along with a stiffener (e.g. hydrated lime) to produce pellets. These pellets are cooled, and a coating is applied to prevent coagulation.

The pelletized binder is marketed under the brand name "PelltPave". In pellet form, the binder is much easier to handle, store and transport. They are delivered to a job site in dry format, and the Pellets can be introduced directly into the mixer at an asphalt plant with no requirement for sophisticated handling, batching or conveyance equipment. Cost analysis conducted by the manufacturer indicates that overall PelletPave mixtures are more economical than comparable polymer modified asphalts e.g. SBS modified HMA [12].

\section{Testing methods}

In the first phase of testing, the neat 60/70pen grade Kuwaiti bitumen (Q8) and the PG6722 Inman bitumen were subjected to PG grading in accordance with AASHTO T315 [13] and Multiple Stress Creep Recovery (MSCR) tests in accordance with AASHTO T350 [14]. An Anton Paar "SmartPave 102" DSR with $25 \mathrm{~mm}$ parallel plate attachment at $1 \mathrm{~mm}$ gap setting was used and specimens were tested at $40^{\circ} \mathrm{C}, 50^{\circ} \mathrm{C}, 60^{\circ} \mathrm{C}$ and $70^{\circ} \mathrm{C}$. DSR testing was carried out on the neat bitumens and the results compared to the same bitumens when modified with $15 \%$ and $18 \%$ crumb rubber content. 
For the MSCR test, a controlled shear stress of $0.1 \mathrm{kPa}$ using a haversine load for $1 \mathrm{~s}$ followed by a $9 \mathrm{~s}$ rest period. For each cycle, the difference between peak strain and final strain is divided by the peak strain to obtain the \% elastic recovery. Twenty creep cycles are applied at $0.1 \mathrm{kPa}$ followed by an additional ten creep-recovery cycles at $3.2 \mathrm{kPa}$. The nonrecoverable creep compliance $\mathrm{J}_{\mathrm{nr}}$ is determined as the ratio of non-recoverable (or permanent) shear strain at the end of the loading cycle by the applied shear stress. The magnitude of $\mathrm{J}_{\mathrm{nr}}$ has been demonstrated to correlate well with rutting performance.

In the second phase of testing, the optimum binder blend (i.e. 18\% rubber) was used in pelletized form (PelletPave) in the manufacture of rubberized hot mix asphalts. Using Kuwait MPW (Type III continuously graded wearing course) HMA gradation as a guideline, three PelletPave contents by mass of asphalt mix were trialled out, namely $2.5 \%$, $3.0 \%$ and $3.5 \%$. The combined gradation of all rubberized-asphalt mixes, comprising of a blend of Gabbro coarse aggregates and crushed sand, natural fine sand and limestone filler was kept constant as shown in Figure 2. At each PelletPave content, 4 binder contents by mass of total mix were trialled, namely $5.0 \%, 5.5 \%, 6.0 \%$ and $6.5 \%$. Binder content is defined simply as the combined mass of neat bitumen plus the mass of PelletPave.

The Marshall method of mix design in accordance with Asphalt Institute MS2 [15] and Kuwait MPW requirements (see Table 4) was used to compact and test the rubberizedasphalt mixes. For this investigation, standard 75 Marshall hammer compaction blows each end of the specimen were adopted, though Ma et al. [16] have highlighted in their investigation the importance of compaction temperatures on rubberized-HMA mixes using the example of a rubber-bitumen blend formulated with $20 \%$ crumb rubber. Using gyratory compaction, Ma et al. [16] demonstrated that the effect of reducing compaction temperature from 180 to $150^{\circ} \mathrm{C}$ caused the air voids content to increase from 4.1 to $7.5 \%$.

\section{Results and analysis}

Current Kuwait MPW specifications encourage the use of polymer modified bitumens including SBS, EVA, Polyethylene, Latex rubber, but does not provide guidance for tyre derived crumb rubber "wet process" modified binders. Nonetheless, any polymer modified bitumen is expected to satisfy AASHTO 315 original binder performance criteria of $\geq 1$ $\mathrm{kPa}$ at $76^{\circ} \mathrm{C}$. The $\mathrm{G}^{*} / \mathrm{sin} \delta$ results shown in Table 1 show that at $15 \%$ crumb rubber content, which is the minimum amount of rubber stipulated by the US Rubber Pavement Association, the Inman modified binder readily exceeds the $76^{\circ} \mathrm{C}$ requirement. Due to unforeseen equipment failure, it was not possible to produce the results for Q8+15\% rubber in time for this paper. At $18 \%$ rubber content, Q8 bitumen satisfies the $\mathrm{G}^{*} / \sin \delta 1 \mathrm{kPa}$ target at $89^{\circ} \mathrm{C}$, whilst the Inman bitumen passes the target at $100^{\circ} \mathrm{C}$.

In general, bitumens with higher $\mathrm{G}^{*} / \sin \delta$ values display greater resistance to rutting because the amount of dissipated energy after each loading cycle, which is related to the appearance of rutting in the pavement, is reduced. This premise is based on the assumption that rutting is a stress-controlled, cyclic loading phenomenon [17].

For the sake of comparison, the $\mathrm{G}^{*} / \mathrm{sin} \delta$ results from another investigation [18], where crumb rubber (passing $1.18 \mathrm{~mm}$, retained on $200 \mu \mathrm{m}$ ) was mixed with 60/70pen grade bitumen at $180^{\circ} \mathrm{C}(2000 \mathrm{rpm})$ for 40 minutes are shown in Figure 2. Kumar [18] reported that as temperature increased, both loss and storage moduli decreased, though the loss modulus was dominant across the entire range of temperatures investigated (i.e. 46 to $82^{\circ} \mathrm{C}$ ), and it was thus concluded that the rubber-bitumen blends (up to a rubber content of $9 \%$ ) showed a predominately viscous behavior ( $\left.G^{\prime}>G^{\prime}\right)$. This is clearly not the case in this investigation when considering the very high MSCR elastic recovery results at $15 \%$ and $18 \%$ crumb rubber content, even at $70^{\circ} \mathrm{C}$, as shown in Table 2. 
Table 1. PG grading results at $10 \mathrm{rad} / \mathrm{s}, 12 \%$ strain.

\begin{tabular}{|c|c|c|c|c|c|c|}
\hline & $\begin{array}{c}\text { Test Temp. } \\
\left({ }^{\circ} \mathbf{C}\right)\end{array}$ & $\begin{array}{c}\text { Shear } \\
\text { Stress } \\
(\mathbf{k P a})\end{array}$ & $\begin{array}{c}\text { Phase } \\
\text { angle } \\
(\mathbf{d e g})\end{array}$ & $\begin{array}{c}\text { Ave. } \mathbf{G}^{*} \\
\mathbf{( k P a )}\end{array}$ & $\begin{array}{c}\mathbf{G}^{*} / \mathbf{s i n} \delta \\
\mathbf{( k P a )}\end{array}$ & $\begin{array}{c}\text { Pass/Fail } \\
\text { temp. } \\
\left({ }^{\circ} \mathbf{C}\right)\end{array}$ \\
\hline Inman & 70 & 0.081 & 88 & 0.7 & 0.68 & Pass 67 \\
\hline Q8 & 76 & 0.065 & 87.7 & 0.5 & 0.54 & Pass 70.7 \\
\hline Inman $+15 \%$ & 100 & 0.082 & 48.6 & 0.7 & 0.91 & Pass 97.9 \\
\hline Inman +18\% & 100 & 0.125 & 54.1 & 1.0 & 1.28 & Pass 100 \\
\hline Q8+18\% & 94 & 0.076 & 51.2 & 0.6 & 0.81 & Pass 89.1 \\
\hline
\end{tabular}

Table 2. Multiple stress creep recovery (MSCR), percent elastic recovery test results.

\begin{tabular}{|c|c|c|c|c|c|c|c|c|}
\hline & \multicolumn{4}{|c|}{$\begin{array}{c}\text { Ave. Recovery at } \mathbf{0 . 1 k P a} \\
(\%)\end{array}$} & \multicolumn{4}{c|}{$\begin{array}{c}\text { Ave. Recovery at } 3.2 \\
(\%)\end{array}$} \\
\hline & $\mathbf{4 0}^{\circ} \mathbf{C}$ & $\mathbf{5 0}^{\circ} \mathbf{C}$ & $\mathbf{6 0}^{\circ} \mathbf{C}$ & $\mathbf{7 0}^{\circ} \mathbf{C}$ & $\mathbf{4 0}^{\circ} \mathbf{C}$ & $\mathbf{5 0}^{\circ} \mathbf{C}$ & $\mathbf{6 0}^{\circ} \mathbf{C}$ & $\mathbf{7 0}^{\circ} \mathbf{C}$ \\
\hline Inman & - & 7.97 & 1.76 & 0 & 0 & 2.08 & 0 & 0 \\
\hline Q8 & 28.43 & 12.52 & 3.36 & 0 & 25.27 & 6.11 & 0 & 0 \\
\hline Inman +15\% & 91.07 & 91.55 & 93.15 & 99.41 & 82.21 & 70.59 & 34.77 & 9.84 \\
\hline Q8+15\% & 92.27 & 98.32 & 99.55 & 99.73 & 76.47 & 57.95 & 22.48 & 5.18 \\
\hline Inman +18\% & 94.92 & 98.56 & 99.12 & 97.6 & 87.53 & 80.4 & 49.05 & 16.72 \\
\hline Q8+18\% & 97.65 & 99.51 & 99.71 & 99.91 & 88.9 & 92.81 & 79.18 & 29.94 \\
\hline
\end{tabular}

Though there are no requirements for the MSCR test within the current Kuwait MPW highway materials specifications, it is currently being trialed on specific motorway projects. Some research indicates that the percent recovery obtained in the MSCR test can directly measure the elastic response of the modified binder, and at $3.2 \mathrm{kPa}$ applied stress, $\mathrm{J}_{\mathrm{nr}}$ values $\leq 2 \mathrm{kPa}^{-1}$ have been recommended as an indication of the recommended level of binder elasticity [17]. Other researchers set a more modest target by proposing that mixes with $\mathrm{J}_{\mathrm{nr}}$ approaching $0.5 \mathrm{kPa}^{-1}$ or lower can be expected to perform well from a binder perspective [19]. With $15 \%$ rubber content, both the Inman and Q8 binders were below $2 \mathrm{kPa}^{-1}$ at $70^{\circ} \mathrm{C}$, whilst at $18 \%$ rubber, the Q8 binder had $\mathrm{J}_{\mathrm{nr}}$ values below $0.5 \mathrm{kPa}^{-1}$, as shown in Table 3 .

Table 4 shows the current MPW specifications for HMA wearing courses, whilst Figure 3 shows the dense aggregate gradation used for all mixes in this investigation. The gradation was designed to be on the finer side of the maximum density line to allow for higher optimum binder contents than the MPW recommended range of 4.0 to $5.5 \%$. The higher binder contents are intended to act as precautionary measures against fatigue cracking and bitumen stripping in wet conditions, whilst the elevated risk of rutting should be safeguarded against by the higher binder stiffness and elastic recovery properties.

Figures 4, 5 and 6 show the air voids, voids filled with bitumen and voids in mineral aggregate values at all binder contents for the $2.5 \%, 3.0 \%$ and $3.5 \%$ PelletPave mixes. Taking all the volumetric and mechanical properties tested thus far into consideration, the optimum binder contents for the 2.5\%, 3.0\% and 3.5\% PelletPave mixes were 5.5\%, 5.7\%, and $5.8 \%$ respectively. At the optimum binder content for each of the three PelletPave proportions, with the exception of the Marshall Stability requirement of $18 \mathrm{kN}$, all other Marshall properties were satisfied. 
Overall, taking into consideration all the volumetric, stability and flow results, though satisfactory, the results did not point to a clear top performing formulation, and until additional more sophisticated mix characterization tests are carried out, e.g. stiffness, wheel tracking, fatigue, it would be difficult to conclude which final mix formulation to adopt for the road trial.

Table 4 shows the current MPW specifications for HMA wearing courses, whilst Figure 3 shows the dense aggregate gradation used for all mixes in this investigation. The gradation was designed to be on the finer side of the maximum density line to allow for higher optimum binder contents than the MPW recommended range of 4.0 to $5.5 \%$. The higher binder contents are intended to act as precautionary measures against fatigue cracking and bitumen stripping in wet conditions, whilst the elevated risk of rutting should be safeguarded against by the higher binder stiffness and elastic recovery properties.

Figures 4, 5 and 6 show the air voids, voids filled with bitumen and voids in mineral aggregate values at all binder contents for the $2.5 \%, 3.0 \%$ and $3.5 \%$ PelletPave mixes. Taking all the volumetric and mechanical properties tested thus far into consideration, the optimum binder contents for the 2.5\%,3.0\% and 3.5\% PelletPave mixes were 5.5\%, 5.7\%, and $5.8 \%$ respectively. At the optimum binder content for each of the three PelletPave proportions, with the exception of the Marshall Stability requirement of $18 \mathrm{kN}$, all other Marshall properties were satisfied.

Overall, taking into consideration all the volumetric, stability and flow results, though satisfactory, the results did not point to a clear top performing formulation, and until additional more sophisticated mix characterization tests are carried out, e.g. stiffness, wheel tracking, fatigue, it would be difficult to conclude which final mix formulation to adopt for the road trial.

\section{Conclusions}

Experimental work carried out at Kuwait Institute for Scientific Research in collaboration with Phoenix Industries USA has shown that Kuwaiti 60/70pen grade bitumen is suitable for modification with waste tyre derived crumb rubber.

Performance grading and multiple stress creep recovery tests conducted using a DSR at a range of test temperatures indicated that at $18 \%$ rubber content, the resultant rubberbitumen blend has excellent rheological characteristics as evidenced by $70^{\circ} \mathrm{C}$ elastic recovery and non-recoverable creep compliance results.

A pelletized version of the best performing rubber-bitumen blend, commercially branded as "PelletPave", was next utilized to substitute part of the total required binder content, in the formulation of rubberized-asphalt mixes using the Marshall method of mix design. Three PelletPave contents were trialed out at $2.5 \%, 3.0 \%$ and $3.5 \%$ by mass of mix, on a single continuously graded wearing course mix gradation and the optimum total binder contents (o.b.c.) were determined at 5.5\%, 5.7\%, and 5.8\% respectively by mass of mix.

Overall, the volumetric properties of all mixes at o.b.c., i.e. voids, voids filled with bitumen and voids in the mineral aggregate were all satisfactory. Stability values were below the target of $18 \mathrm{kN}$ and the results generated thus far were insufficient to aid in the selection of the best performing formulation. Additional, more detailed testing is required to better characterize the mixes rheological response including creep and fatigue properties. 
Table 3. Multiple stress creep recovery (MSCR), non-recoverable creep compliance $\left(\mathrm{J}_{\mathrm{nr}}\right)$ results.

\begin{tabular}{|c|c|c|c|c|c|c|c|c|}
\hline & \multicolumn{4}{|c|}{$\begin{array}{c}\text { Ave. Jnr at 0.1 kPa } \\
(\mathbf{1} / \mathbf{k P a})\end{array}$} & \multicolumn{4}{c|}{$\begin{array}{c}\text { Ave. Jnr at 3.2 kPa } \\
\mathbf{( 1 / k P a}\end{array}$} \\
\hline & $\mathbf{4 0}^{\circ} \mathbf{C}$ & $\mathbf{5 0}^{\circ} \mathbf{C}$ & $\mathbf{6 0}^{\circ} \mathbf{C}$ & $\mathbf{7 0}^{\circ} \mathbf{C}$ & $\mathbf{4 0}^{\circ} \mathbf{C}$ & $\mathbf{5 0}^{\circ} \mathbf{C}$ & $\mathbf{6 0}^{\circ} \mathbf{C}$ & $\mathbf{7 0}^{\circ} \mathbf{C}$ \\
\hline Inman & - & 0.8532 & 3.9024 & 14.4025 & 0 & 0.945 & 4.3377 & 15.7782 \\
\hline Q8 & 0.0862 & 0.5366 & 2.455 & 9.0424 & 0.0899 & 0.5926 & 2.765 & 10.1493 \\
\hline Inman +15\% & 0.004 & 0.0129 & 0.0297 & 0.0055 & 0.0082 & 0.0456 & 0.3521 & 1.6916 \\
\hline Q8+15\% & 0.0023 & 0.0018 & 0.0014 & 0.0015 & 0.0072 & 0.0477 & 0.3401 & 1.5496 \\
\hline Inman +18\% & 0.0015 & 0.0012 & 0.002 & 0.0118 & 0.0036 & 0.017 & 0.1429 & 0.8841 \\
\hline Q8+18\% & 0.0005 & 0.0003 & 0.0005 & 0.0002 & 0.0024 & 0.0049 & 0.0399 & 0.443 \\
\hline
\end{tabular}

Table 4. Kuwait MPW Marshall design criteria for Type III wearing course asphalt concrete mix.

\begin{tabular}{|l|c|c|}
\hline & Min. & Max. \\
\hline No. of compaction blows each end of specimen & & 75 \\
\hline Stability (N) & 18000 & - \\
\hline Flow (0.25mm) & 8 & 16 \\
\hline VMA & 14 & - \\
\hline Air Voids (\%) & 4 & 6 \\
\hline Aggregate Voids Filled with Bitumen (\%) & 63 & 75 \\
\hline
\end{tabular}

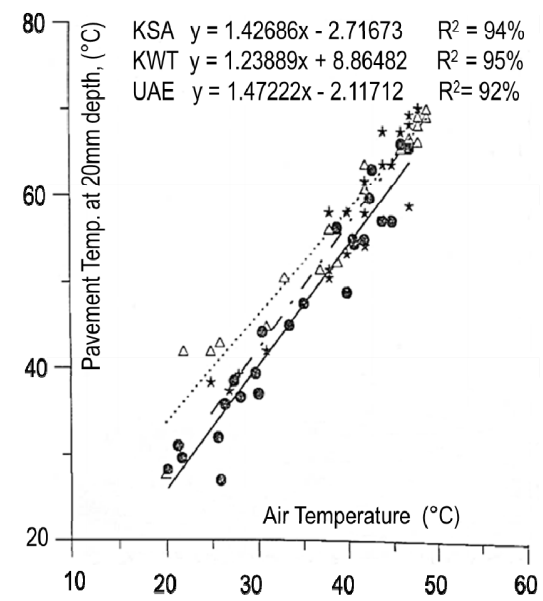

Fig. 1. Relationship between air temperature and pavement temperature at a depth of $20 \mathrm{~mm}$. KSA $=$ Kingdom of Saudi Arabia, KWT $=$ Kuwait, UAE = United Arab Emirates [1, 2].

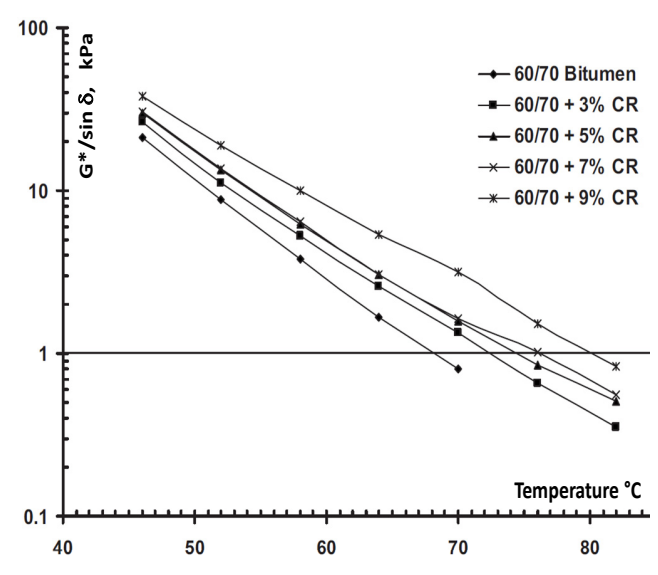

Fig. 2. $\mathrm{G}^{*} / \mathrm{sin} \delta$ measured at $10 \mathrm{rad} / \mathrm{s}$ as a function of temperature for 60/70pen bitumen modified with crumb-rubber [18]. 


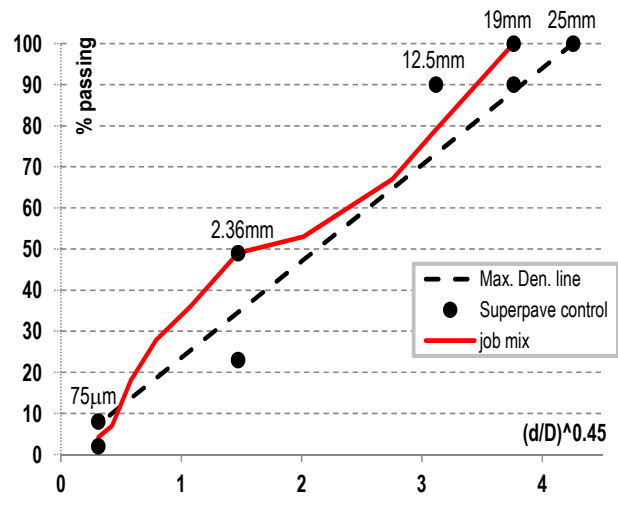

Fig. 3. Aggregate gradation used for production of rubberized-HMA mixes in this investigation

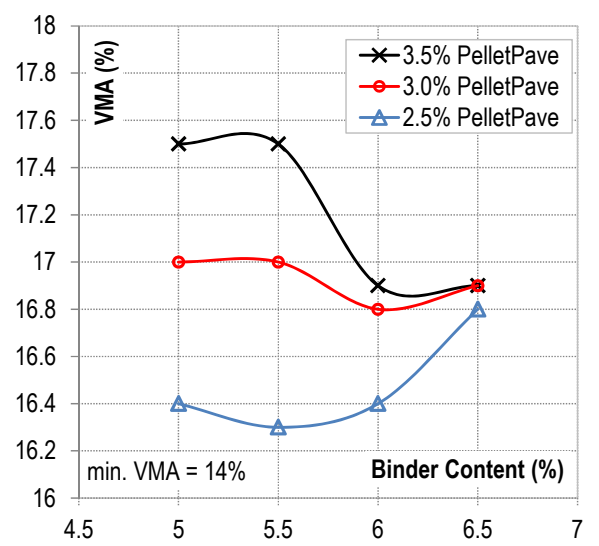

Fig. 5. VMA versus binder content for $2.5 \%$, $3.0 \%$ and $3.5 \%$ PellePave HMA mixes.

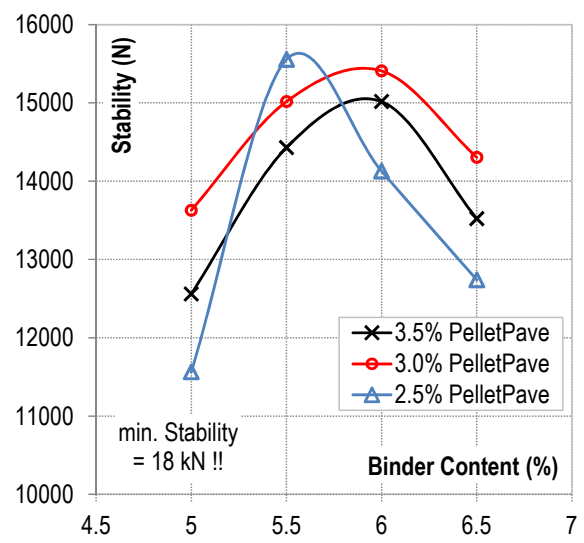

Fig. 7. Marshall Stability versus binder content for $2.5 \%, 3.0 \%$ and $3.5 \%$ PellePave HMA mixes.

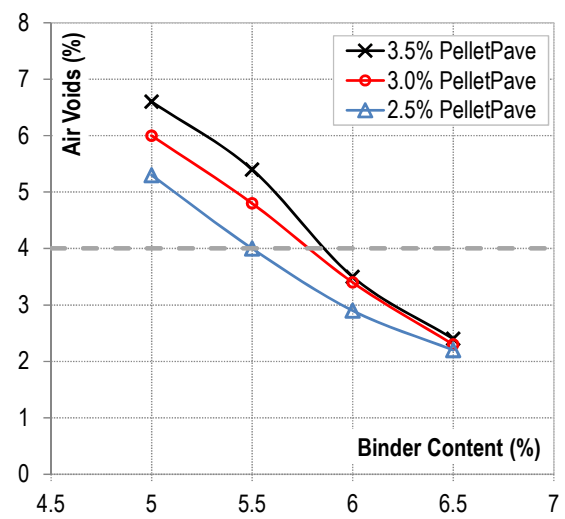

Fig. 4. Air voids versus binder content for $2.5 \%, 3.0 \%$ and $3.5 \%$ PellePave HMA mixes.

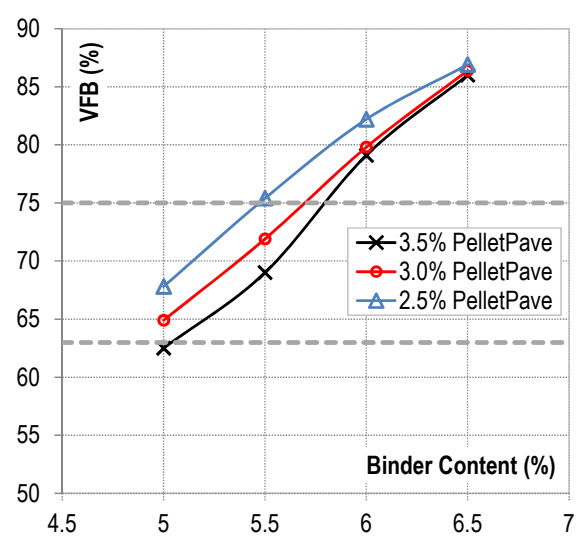

Fig. 6. VFB versus binder content for $2.5 \%$, $3.0 \%$ and $3.5 \%$ PellePave HMA mixes.

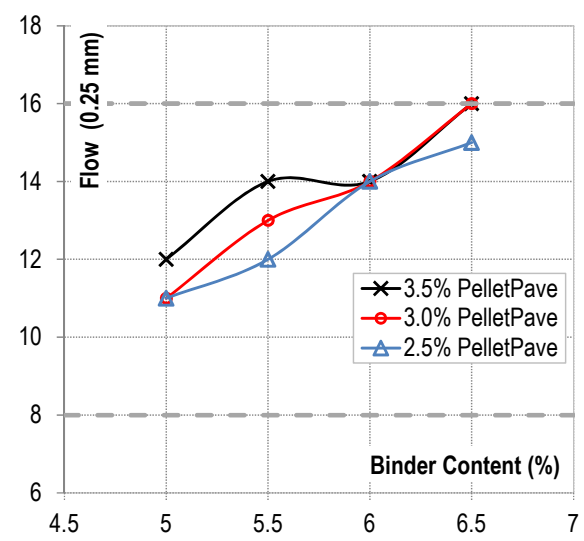

Fig. 8. Flow versus binder content for $2.5 \%$, $3.0 \%$ and $3.5 \%$ PellePave HMA mixes. 


\section{References}

1. H.K. Al-Abdul Wahhab, I.M. Asi, I.A. Dubabe and M.F. Ali, Development of performance-based bitumen specifications for the Gulf countries, Construction and Building Materials, Vol. 11, pp. 15-22 (1997).

2. F. Bissada, Asphalt Pavement Temperature Related to Kuwait Climate, HRR 404, Transportation Research Board, Washington D.C., pp. 71-85 (1972).

3. M. Cope. Performance and Economic Benefits of Paving with Asphalt Rubber; www.ra-foundation.org: Rubber Asphalt Foundation (1985).

4. Sacramento County Department of Environmental Review and Assessment, Report on the Status of Rubberized Asphalt - Traffic Noise Reduction in Sacramento County, Nov. (1999).

5. J. D'Angelo, Rubberized Asphalt Foundation Study Concludes Recycled Tire Rubber Can Take Place of Performance Grade Polymer Modified Asphalt. A whitepaper issued by the Rubberized Asphalt Foundation (2013).

6. D. Lo Presti, Recycled Tyre Modified Bitumens for road asphalt mixtures: A literature review, Construction and Building Materials, Vol. 49, pp. 863-881 (2013).

7. G. Way, K. Kaloush, and K. Biligiri, Consulpav Int. and Arizona State University. Asphalt-Rubber Standard Practice Guide. Rubber Pavement Association (2012).

8. M. Stroup-Gardiner, Cost Factors, Federal Highway Administration, Crumb Rubber Modifier Workshop, Denver, Colorado, March (1993).

9. G.R. Morris, K.R. Hansen, Cost Considerations of Asphalt-Rubber Hot Mix, www.rubberpavements.org/ARTIC/Cost/ARTIC_Cost_RPA_A1595.html (1993).

10. B. Bairgi, S.M. Hossain, R. D. Henrix, Investigation of rheological properties of asphalt rubber toward sustainable use of scrap tires, IFCEE 2015, ASCE, pp. 359-368 (2015).

11. Phoenix Industries, LLC, 1988. 300 E. Delhi Ave. N. Las Vegas, Nevada, 89032, USA. www.PhoenixIndustries.com (1988).

12. S.N. Amirkhanian, F. Xiao and K. Sockwell, Performance properties of polymer modified pelletized asphalt mixtures, Proc. of the 2015 Int. Airfield and Highway Pavements Conference, ASCE, pp. 120-130 (2015).

13. AASHTO T 315-12 (R2016), Standard method of test for determining the rheological properties of asphalt binder using a dynamic shear rheometer (DSR).

14. AASHTO T 350-14, Standard method of test for multiple stress creep recovery (MSCR) test of asphalt binder using a dynamic shear rheometer (DSR).

15. Asphalt Institute, MS-2 Asphalt Mix Design Methods, ISBN: 9781934154700.

16. T. Ma, H. Wang, L. He, Y. Zhao, X. Huang and J. Chen, Property characterization of asphalt binders and mixtures modified by different crumb rubbers, J. Mater. Civ. Eng., Vol. 29, Issue 7 (2017).

17. M.D. Domingos, A.L. Faxina, Susceptibilty of asphalt binders to rutting: literature review, J. Mater. Civ. Eng., Vol. 28, Issue 2, (2016).

18. P. Kumar, H.C. Mehndiratta, K. Singh, Comparative study of rheological behavior of modified binders for high-temperature areas, Journal of Materials in Civil Engineering, Vol. 22, Issue 10 (2010).

19. E. Dubois, Y. Mehta, A. Nolan, Correlation between multiple stress creep recovery (MSCR) results and polymer modification of binder, Construction and Building Materials, Vol. 65, pp. 184-190 (2014). 